\title{
Combination Vaccines
}

\author{
Col SK Jatana*, Brig MNG Nair"
}

\begin{abstract}
Infections are still a leading cause of morbidity and mortality in children under five years of age, most of which can be prevented by vaccination. However, there are too many vaccines to be administered, increasing the cost of immunization and visits to the paediatrician. Combination vaccines can be an answer to these problems till the development of a single vaccine containing all the possible antigens. Researchers are aiming at development of an ideal vaccine, which can be given orally at birth, has negligible side effects, is heat stable and is affordable to all the parents.
\end{abstract}

MJAFI 2007; 63 : 167-171

Key Words: Infections; Immunization; Combination vaccines

\section{Introduction}

$\mathrm{V}$ accination as a deliberate attempt to protect human beings against disease has a long history and more widespread use of vaccines could prevent 1.6 million deaths a year, among children less than five years of age. Over the next few years a new generation of vaccines will become available that could save the lives of up to 10 million individuals: e.g. vaccines against diarrhoeal diseases, hepatitis $\mathrm{C}$, malaria, acquired immunodeficiency syndrome (AIDS), sexually transmitted and other diseases [1]. Current development efforts seek combination vaccines that protect against multiple pathogens, in keeping with the ultimate goal of combining all the antigens recommended for routine immunization into a single multivalent product [2].

Combining multiple related or unrelated antigens into a single vaccine is not a new concept and the first combination vaccine licensed in United States of America was trivalent influenza in 1945. Diphtheria, Pertussis, Tetanus (DPT) vaccine although developed in 1943, was not licensed till 1948.Efforts to overcome the interference seen with simultaneous administration of three live vaccines delayed the licensing of trivalent Oral Polio Vaccine (OPV) till 1963. Measles, Mumps, Rubella (MMR) was licensed in 1971 and quadrivalent meningococcal vaccine in 1978 [2].

Growth in the number of effective vaccines has posed substantial economic and logistical difficulties. In 1990 the Children's Vaccine Initiative (CVI) was launched at the World Summit for Children in New York City. The CVI proposed that the ideal vaccine would provide all indicated antigens in a single dose (preferably oral), would be heat stable, effective when administered soon after birth and affordable [3]. Combination vaccines [4], offer the benefits of decreased number of injections, decreased number of visits, decreased cost of administration, increased compliance, ease of storage and improved record keeping and tracking.

\section{Challenges in the Development of Combination Vaccines}

The development of new vaccines brings new challenges at the manufacturing, trial and administration stages. Combining multiple antigens into one injection requires demonstration that the combination will not materially reduce the safety or immunogenecity of the component vaccines. Combination vaccine trials should be prospective, randomised, double blinded and should have control (comparison) groups. Identifying the control groups could be problematic when multicomponent vaccine is evaluated. Other factors like sequence of administration of certain antigens may play an important role in immunogenecity [4-6].

\section{Immunological Interference}

Immunologic interaction between components of a combination vaccine can occur leading to immunological interference and the three major mechanisms are:

a. Antigen Competition: The interaction can enhance the immune response to individual components as occurs in whole cell pertussis vaccine when combined with diphtheria toxoid. Usually combination of vaccines results in no effect or a depression of immune response to one or more vaccine component [7-8]. 
b. Carrier Induced Epitope Expression: It is an immunologic phenomenon relevant to combination vaccines, antibody responses to hapten polysaccharide vaccine (e.g. $H$ influenzae $b$ ) presented on a carrier protein (e.g. tetanus toxoid, diphtheria toxoid) are inhibited by prior immunization with the specific carrier [9-10].

c. Induction of Interferon: Combination live vaccines can interfere immunologically with each other e.g. one vaccine may stimulate interferon production that may inhibit replication of another virus [4].

Chemical or physical interactions among the vaccine components being combined can result in an alteration of the immune response to vaccine [7]. The combination of one antigen that is administered with adjuvant with an antigen that is not administered with adjuvant, may lead to displacement of the adjuvant and reduced immunogenecity of the first vaccine. Also, the adjuvant may combine with the second antigen and may alter the response of second antigen as well. Similarly buffers, stabilizers and similar components may interfere with the components of the other vaccine. Such vaccines cannot be mixed in the vial and separation of the two vaccines in a dual-chambered syringe can circumvent this problem. However with proper mixing procedures, the uniformity and stability of various components of a combination vaccine may not be an issue during its production [11].

Ethical concern have to be taken into account because vaccines administered routinely cannot be withheld in order to study vaccine interactions [2]. Multiple vaccine preparations against the same pathogen are also available, which makes the studies more complex. For example, there are three $H$ influenza $b$ (Hib) conjugate vaccines, two hepatitis $B$ vaccines and seven DPT vaccines available for use in infancy and as many as 42 DTaP/Hib/HB combination vaccines have to be studied.

The other requirements are that the product should be stable, each component of the combination vaccine should be given at the same age, local and systemic adverse effects should be minimal, immune response to each component should be satisfactory and the requirement of booster for each component should be similar.The combining of various antigens can be performed in many ways [4]. At manufacturing site the first approach is using multiple different antigens of an organism that have been mixed together as a single product to immunize against multiple serotypes (strains) causing disease e.g. polio (3 strains), 23/7 valent pneumococcal. The second approach brings together antigen from pathogens causing different diseases e.g. DPT. Vaccines can be mixed from separate vials at the time of administration or different vaccines can be placed in separate chambers of a multi chambered syringe. Recent development of biodegradable microspheres, represent a potential tool for the delivery of combination vaccines. Childhood and malaria vaccines combined in biodegradable microspheres produce immunity with synergistic interactions. However, more studies and research is required before this technique is commercially available [12].

\section{Traditional Combined Vaccines}

Diphtheria, Pertussis, Tetanus (DPT), Measles, Mumps, Rubella (MMR), Injectable Polio Vaccine (IPV) and Oral Polio Vaccine (OPV) are in use successfully for many years. In the last few years more antigens have been added to these traditional vaccines.

1. DPT/IPV: Interest in combining DPT/IPV was generated when enhanced potency IPV became available thus eliminating the necessity of frozen shipments for OPV. In addition administration of IPV would eliminate the risk of vaccine-associated polio. Antibody responses to pertussis and poliovirus components may be substantially reduced in combination than when given alone. However, poliovirus seroconversion rates and absolute antibody levels remained high in combined vaccines $[13,14]$.

2. DPT/Conjugate (Hib) : Various studies have compared DPT combinations with unconjugated Polyribosyl phosphate (PRP) or conjugated PRP Hib vaccine. The surveillance data provides evidence that use of combined DPT/Conjugate Hib does not reduce efficacy in comparison to DPT and conjugate Hib given separately $[8,15]$.

3. DPT/Hepatitis B and DPT/Hepatitis B/Hib: A number of studies have evaluated these combination vaccines and results are variable. In general, the groups with lower antibody responses still attained levels considered protective. One study evaluated the effect of booster dose of DPT/Hepatitis B/Hib given to subjects who received DPT/Hepatitis B/ Hib for the primary series. The group had high antibody response and mean levels were higher in the group primed with DPT/Hepatitis B/Hib, especially with PRP (Hib) [16-17].

4. MMR/Varicella: Initial studies on MMRV vaccine produced showed low seroconversion rates for varicella, but latest formulations show uniformly high rates of seroconversion. Studies carried out after one year of immunization compare favourably with combined and separate vaccine groups. Adverse reactions in all studies were same except that morbilliform rash was more common in combined 
vaccine [18].

\section{Combinations with DTaP (Acellular Pertussis)}

1. DTaP/HB: This combination vaccine retains the immunogenecity and safety profiles of the separate components and delivers good antibody concentrations at a variety of schedules. A comparison of combination vaccine at 2, 4, 6 months versus the currently recommended schedules-HB at birth, $1 \& 6$ months and DPT at $6,10 \& 14$ weeks, found similar or higher antibody responses for combined vaccine for every component, which was significantly lower [19]. However, the mean HB antibody levels were high and $98 \%$ of subjects had levels greater than $10 \mathrm{mIU} / \mathrm{mL}$, which are considered protective.

2. DTaP/Hib: Studies conducted in United States and Taiwan have evaluated a variety of DTaP/Hib combinations and similar results obtained [20,21]. All combinations are highly immunogenic when used to boost previously primed children. In primary immunization, DTaP responses have been same but response to Hib in combination vaccines has been substantially lower than that obtained when it is given separately. However, the immune response to a booster dose of Hib conjugate vaccine indicated that the primary dose has effectively stimulated the immune response. A study conducted in Germany has shown that vaccine effectiveness of combination vaccines against invasive Hib infection was $89.6 \%$ (95\% CI 67.0-96.7) for an incomplete primary series, $96.7 \%$ (95\% CI 87.7-99.1) for a full primary series and 98.5\% (95\% CI 94.5-99.6) for a booster dose irrespective of the fact whether the primary immunization was done with a monovalent or a combination vaccine [22].

3. DTaP/HB/Hib: A number of studies were conducted evaluating DTaP/HB/PRP-T combination vaccine. Infants given a combined DTaP/ HepB/PRP-T vaccine experienced a significantly lower antibody response to the PRP-T component than infants given PRP-T vaccine as a separate injection. But after the booster dose the antibody levels were comparable and protective, signifying that the combination vaccine had successfully primed the immune system [20,21].

4. DTaP/HB /IPV and DTaP/HB/ Hib/IPV : Similar findings were observed in a few studies and only PRP response was lower in combination vaccine as compared to separate vaccines but sufficient to protect against the disease [23, 24]. The DPTaHBV-IPV/Hib (hexavalent) combined vaccine is protective and produces adequate immunity when administered at 3,5 and 11 month of age and is a good alternative to separate administration of monovalent Hib vaccine and the pentavalent DTaPHBV-IPV combination vaccine [25].

\section{Combination with $\mathrm{HB}$ without DPT}

1. HB/Hib : A study comparing combination vaccine and separate vaccines given at 2, 4, 6 and booster between 12 and 15 months of age has shown [26] that antibody response to $\mathrm{Hib}$, were $72 \%$ and $76 \%$ at 6 months, increasing to $92 \%$ and $93 \%$ after booster dose with combination and separate vaccines respectively. Responses to HB component were also comparable.

2. HB/Hepatitis A: In a US trial, a combination hepatitis vaccine on a $0,1,6$ month schedule or corresponding monovalent vaccines (Havrix, 1440 EL.U/ml of hepatitis A antigen at 0, 6 months and Engerix-B, 20 mcg of hepatitis B surface antigen at $0,1,6$ months) were administered concurrently to 773 adults. The results showed that both the schedules were safe and the level of immunity produced was comparable in the two groups [27]. However this combination can be administered after one year of age in children who have not been immunized earlier.

3. Hep A/Typhoid: New Hepatitis A/Typhoid combined vaccine as well as traditional vaccine induced high levels of protective antibodies [28], but the combined vaccine has higher incidence of local reactions.

Besides DPT, OPV and MMR, DPT + HepB, $\mathrm{DPT}+\mathrm{Hib}, \mathrm{DPT}+\mathrm{HepB}+\mathrm{Hib}$ and HepA+HepB are available in India. Internationally instead of whole cell pertussis antigen in DPT vaccine, combinations with acellular pertussis (aP) and $\mathrm{DTaP}+\mathrm{HB}+\mathrm{IPV}$ and $\mathrm{DTaP}+\mathrm{HB}+\mathrm{Hib}+\mathrm{IPV}$ are also available.

The cost of combination vaccines is higher than the combined cost of the individual vaccines. The Indian Academy of Paediatrics recommends that combination vaccines should replace individual vaccines wherever feasible and the dosing schedule can be modified accordingly e.g. the schedule of DPT can be changed to 2, 4 and 6 months if a combination of DPT and Hib has to be administered. All infants should receive the $1^{\text {st }}$ dose of Hep B vaccine soon after birth and before hospital discharge (only monovalent vaccine to be used). The $1^{\text {st }}$ dose may be administered by age of 2 months if mother is Hepatitis B negative. Here combination vaccine (DPT $+\mathrm{HepB}+\mathrm{Hib}$ ) can be used. The second dose of Hep B should be administered at least four weeks after the first dose and third dose given at least 16 weeks after the first dose. The recommended schedule of 
Table 1

\section{Recommended Childhood and Adolescent Immunization Schedule UNITED STATES • 2005}

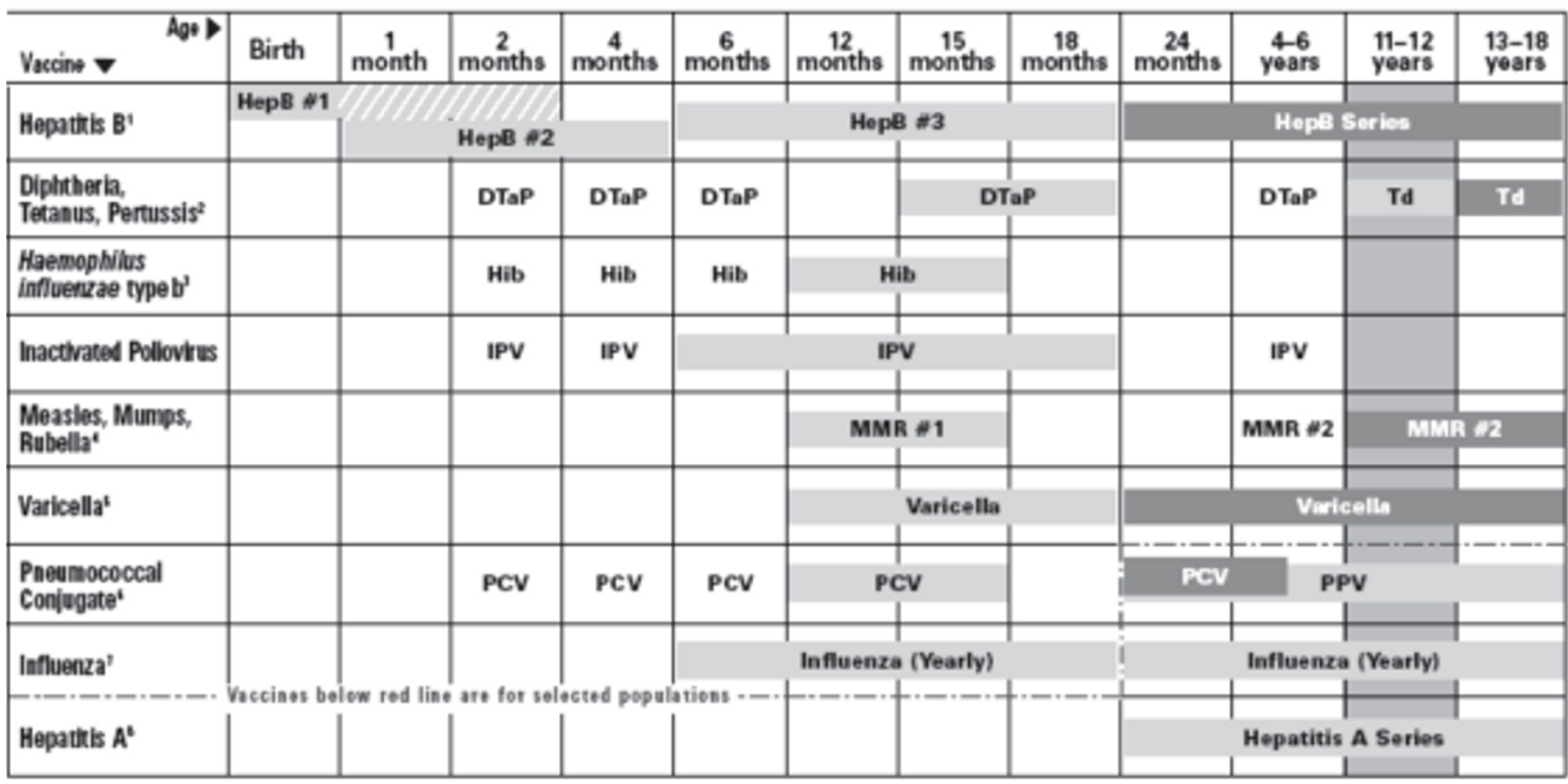

This schedule indicates the accommended ages for routine administation of curnenth Foensed childhood vaccines, as of Dscenber 1, 2004, for childen through age 18 yerrs. Arry dose aot administared at the recommended age should be administered at any subsequent visit whon indicatod and feasible.

Indicates age eroups that wartant soecial effort to administer those viccines not previously adninistared. Add tibnal vaccines may be licensed and reconmended dring the yeas. Licensed combinaton vacines may be used whenever any components of the combination an indicated and other components of the vaccine are not contraindicated. Providers stould coustit the manufacturers' package inserts foe detailed recommendrtions. Clinically significant advesse everts that follow immunication should be reported to the Vaccine Acterse Eveat Raporting System NAERSi. Guidance about how to obtain and complets a VAERS foem is avalatie at www.vaers.org or by tolsphoae, 800-822-7057.

Range of recommended apes Preadolescent assessment
Only if mother HBsApl-)

Catch-up immunization immunizations in the U.S.A is given in Table 1 [29].

Other combination vaccines for consideration especially in the tropical countries include measlesyellow fever, measles-Japanese encephalitis or pertussisbased paediatric combination rabies vaccine. However, the necessity and feasibility of these combinations has to be considered [30].

To conclude, an ideal combination vaccine should provide all antigens in single dose, preferably by the oral route, should be heat stable, should be effective when administered after birth and affordable for all families.

\section{Conflicts of Interest}

None identified

\section{References}

1. Future Vaccine Strategies. www.worldwidevaccines.com

2. Decker MD, Edwards KM. Combination vaccines. In: Vaccines; Plotkin SA, Orenstein WA, editors. $3^{\text {rd }}$ ed. Philadelphia: WB Saunders, 1999: 508-30.

3. Children's Vaccine Initiative [news]. World Health Forum 1992; 13:93.

4. Pichichero ME. New combination vaccines. PCNA 2000; 47: 395-406.

5. Edwards KM, Decker MD. Combination Vaccines: Hopes and challenges. Pediatr Infect Dis J 1994; 13:345-7.
6. Decker MD, Edwards KM. Issues in design of clinical trials of combination vaccines. In: Williams JC, Goldenthal KL, Burns DL, Lewis Jr, editors. Combined Vaccines and simultaneous administration: Current Issues and Perspectives. Ann NY Acad Sci 1995; 754:234-40.

7. Insel RA. Potential alterations in immunogenecity in combining or simultaneously administering vaccine components. Ann N Y Acad Sci 1995; 754:35.

8. Halperin SA, King J, Law B, et al. Safety and immunogenecity of $H$ influenza-tetanus toxoid conjugate vaccine given separately or in combination with a three component acellular pertussis vaccine combined with diphtheria and tetanus toxoid and inactivated poliovirus vaccine for the first four doses. Clin Infect Dis 1999; 28 : 995-1001.

9. Barington T, Gyhrs A, Kristensen K, Heilmann C. Opposite effects of actively and passively acquired immunity to the carrier on responses of human infants to a Hemophilus influenzae type b conjugate vaccine. Infect Immun 1994; 62:9-14.

10. Dagan R, Eskola J, LeClerc C, et al. Reduced response to multiple vaccines sharing common protein epitopes that are administered simultaneously to infants. Infect Immun 1998; 66:2093-8.

11. Morefield GL,HogenEsch H, Robinson JP, Hem SL.Distribution of adsorbed antigen in mono-valent and combination vaccines. Vaccine 2004; 22: 1973-84.

12. Childhood and malaria vaccines combined in biodegradable microspheres produce immunity with synergistic interactions. Peyre M, Audran R, Estevez F, Corradin G, Gander B, Sesardic 
D, Johansen P. J Control Release 2004 ; 99:345-55.

13. Halperin SA, LangleyJM, Eastwood BJ. Effect of inactivated poliovirus vaccine on antibody response to Bordetella pertussis antigens when combined with diphtheria-pertussis-tetanus vaccine. Clin Infect Dis 1996; 22: 59-62.

14. Meschievitz C, Blatter M, Starr S, Fritzell B. Safety and immunogenecity of IPV only or a sequential schedule of IPV (given separately or in combination with DPT) followed by OPV (abstract H089). Abstracts of the $36^{\text {th }}$ Interscience Conference on Antimicrobial agents and Chemotherapy. American Society for Microbiology; Washington DC; 1996.

15. Paradiso PR. Combination vaccines for diphtheria, pertussis, tetanus and Hemophilus influenzae type b. Ann N Y Acad Sci 1995; 754:108-13.

16. Win KM, Aye M, Htay-Htay H, et al. Comparison of separate and mixed administration of DPTw-HBV and Hib vaccines: immunogenecity and reactogenecity profiles. Int $\mathrm{J}$ Infect Dis 1997; 2:79-84.

17. Aristegui J, Garrote E, Gonzalez A, et al. Immune response to a combined hepatitis $\mathrm{B}$, diphtheria, tetanus and whole cell pertussis vaccine administered to infants at 2, 4 and 6 months of age. Vaccine 1997; 15:7-9.

18. White CJ, Stinson D, Staeble B, et al. Measles, mumps, rubella and varicella combination vaccine: safety and immunogenecity alone and in combination with other vaccines given to children. Clin Infect Dis 1997; 24:925-31.

19. Greenberg DP, Wong VK, Partridge S, Howe BJ, Ward JI. Safety and immunogenicity of a combination diphtheria-tetanus toxoids-acellular pertussis-hepatitis B vaccine administered at two, four and six months of age compared with monovalent hepatitis B vaccine administered at birth, one month and six months of age. Pediatr Infect Dis J 2002; 21: 769-77.

20. Lee CY, Thipphawong J, Huang LM, Lee PI, Chiu HH, Lin W, et al. An evaluation of the safety and immunogenicity of a fivecomponent acellular pertussis, diphtheria, and tetanus toxoid vaccine (DTaP) when combined with a Haemophilus influenzae type b-tetanus toxoid conjugate vaccine (PRP-T) in Taiwanese infants. Pediatrics 1999;103:25-30.

21. Greenberg DP, Wong VK, Partridge S, Chang SJ, Jing J, Howe BJ, Ward JI. Immunogenicity of a Haemophilus influenzae type b-tetanus toxoid conjugate vaccine when mixed with a diphtheria-tetanus-acellular pertussis-hepatitis B combination vaccine Pediatr Infect Dis J 2000;19:1135-40.

22. Kalies H, Verstraeten T, Grote V, Meyer N, Siedler A, Schmitt HJ, et al. Four and one-half-year follow-up of the effectiveness of diphtheria-tetanus toxoids-acellular pertussis/Haemophilus influenzae type $b$ and diphtheria-tetanus toxoids-acellular pertussis-inactivated poliovirus/H influenzae type $b$ combination vaccines in Germany. Pediatr Infect Dis J 2004;23:944-50.

23. Yeh SH, Ward JI, Partridge S, Marcy SM, Lee H, Jing J, et al. Safety and immunogenicity of a pentavalent diphtheria, tetanus, pertussis, hepatitis B and polio combination vaccine in infants. Pediatr Infect Dis J 2001;20:973-80.

24. Curran M, Goa K. DPTa-HBV-IPV/Hib Vaccine (Infanrix hexa trade mark ). Drugs 2003;63:673-82.

25. Gabutti G, Zepp F, Schuerman L, Dentico P, Bamfi F, Soncini $\mathrm{R}$, et al. Evaluation of the immunogenicity and reactogenicity of a DPTa-HBV-IPV Combination vaccine co-administered with a Hib conjugate vaccine either as a single injection of a hexavalent combination or as two separate injections at 3,5 and 11 months of age. Scand J Infect Dis 2004;36:585-92.

26. West DJ, Hesley TM, Jonas LC, et al. Safety and immunogenecity of a bivalent Hemophilus influenzae type b / hepatitis B vaccine in healthy infants. Pediatr Infect Dis J 1997; 16:593-99.

27. Joines RW, Blatter M, Abraham B, Xie F, De Clercq N, Baine $\mathrm{Y}$, et al. A prospective, randomized, comparative US trial of a combination hepatitis A and B vaccine (Twinrix) with corresponding monovalent vaccines (Havrix and Engerix-B) in adults. Vaccine 2001; 14;19:4710-9.

28. Beeching NJ, Clarke PD, Kitchin NR, Pirmohamed J, Veitch K, Weber F. Comparison of two combined vaccines against typhoid fever and hepatitis A in healthy adults. Vaccine 2004;15;23:2935.

29. Recommended Childhood and Adolescent Immunization schedule USA2005. www.cdc.gov/nip/recs/child-schedule.

30. Fletcher MA, Fabre P, Debois H, Saliou. Vaccines administered simultaneously: directions for new combination vaccines based on an historical review of the literature. P Int $\mathbf{J}$ Infect Dis 2004;8:328-38.

\section{ATTENTIONADVERTISERS}

Rates of advertisements in Medical Journal Armed Forces India :

\begin{tabular}{|c|c|c|c|}
\hline & Black \& White & Colour & Size \\
\hline Full page (per issue) & Rs. 5000/- & Rs. 10000/- & $25 \times 18 \mathrm{cms}$ \\
\hline \multirow[t]{2}{*}{ Half page (per issue) } & Rs. 3000/- & Rs. 6000/- & $18 \times 12 \mathrm{cms}$ \\
\hline & \multicolumn{3}{|c|}{ (Positive for advertisement to be supplied by client) } \\
\hline \multirow[t]{4}{*}{ Inserts (two pages) } & \multicolumn{3}{|c|}{ Rs. 5000/- per insert (Black and White / Colour) } \\
\hline & \multicolumn{3}{|c|}{ (Printed inserts to be supplied by client) } \\
\hline & \multicolumn{3}{|c|}{ Address for Correspondence } \\
\hline & & AL JOURNAL ARMED FORCES INDIA & \\
\hline
\end{tabular}

